\title{
Partial Escape Width for Nuclei with Neutron Excess
}

\author{
T. N. Leite ${ }^{1}$ and N. Teruya ${ }^{2}$ \\ ${ }^{1}$ Fundação Universidade Federal do Vale do São Francisco (UNIVASF) \\ Colegiado de Engenharia Civil, Juazeiro, BA, Brazil. \\ ${ }^{2}$ Departamento de Física, Universidade Federal da Paraíba \\ CP 5008, 58051-970 João Pessoa, PB, Brazil
}

Received on 8 December, 2004

\begin{abstract}
In this work, the escape width for nuclei with neutron excess is calculated by continuum Random Phase Approximation $(R P A)$ with a careful treatment on the differences between the densities of neutrons and protons.
\end{abstract}

\section{INTRODUCTION}

In this work, the escape width for nuclei with neutron excess is calculated by continuum Random Phase Approximation (RPA) of the Ref.[1] with a careful treatment on the differences between the densities of neutrons and protons [2]. The calculation of partial escape width for each single-hole gives a good estimate of the escape width composition for the population of several single-holes in the residual nucleus. We have applied this method in order to estimate the energy location and the partial escape widths for pygmy dipole resonance $(P D R)$ in the exotic nucleus ${ }^{60} \mathrm{Ca}$ and for isoscalar giant dipole resonance $(I S G D R)$ in the ${ }^{208} \mathrm{~Pb}$.

In the Sect. II of this work, we presented a summary of the continuum RPA formalism that accommodates the differences between neutron and proton densities. We present and comment our results in the Sect. III.

\section{RPA FORMALISM}

The continuum effects in our microscopic calculations are taken into account through a discrete particle-hole basis which accomodates the single-particle resonance widths, resulting in a diagonalization of RPA-like complex matrixes of standard size [1]. The excited states are implemented in the particlehole excitation space, and the open channels correspond to unbound particle-hole states with complex energies, of which the imaginary parts give the single-particle escape widths.

The complex particle-hole modes are solved by a diagonalization of the discrete $R P A$ equations:

$$
\left(\begin{array}{cc}
A & B \\
-B & -A
\end{array}\right)\left(\begin{array}{c}
X^{n} \\
Y^{n}
\end{array}\right)=\widehat{\varepsilon}_{n}\left(\begin{array}{c}
X^{n} \\
Y^{n}
\end{array}\right)
$$

where:

$$
A_{p h p^{\prime} h^{\prime}}=\left(\widehat{\varepsilon}_{p}-\varepsilon_{h}\right) \delta_{p p^{\prime}} \delta_{h h^{\prime}}+V_{p h^{\prime} h p^{\prime}} ; B_{p h p^{\prime} h^{\prime}}=V_{p p^{\prime} h h^{\prime}} ;
$$

and $\widehat{\varepsilon}_{p}\left(\widehat{\varepsilon}_{p}=\varepsilon_{p}-\frac{1}{2} i \Gamma_{p}\right)$ are the complex energies of the single-particle resonances [3, 4]. Diagonalizing the complex Eq.(1), we have the complex eigenvectors given by complex $X_{p h}^{n}$ and $Y_{p h}^{n}$ amplitudes and complex eigenvalues $\widehat{\varepsilon}_{n}=$ $\varepsilon_{n}-\frac{1}{2} i \Gamma_{n}^{\uparrow}$. Thus, in this discrete particle-hole subspace, the
TABLE I: The evaluated escape widths, and the estimates of the partial escape widths for each single-hole, of the three main peaks that compose the $P D R$ in $\sim 8.6 \mathrm{MeV}$.

\begin{tabular}{l|c|c|c}
\hline \hline$\varepsilon_{n}-\frac{1}{2} i \Gamma_{n}^{\uparrow}(\mathrm{MeV})$ & $8.05-i 1.29$ & $8.69-i 1.35$ & $8.94-i 0.40$ \\
\hline single-hole & \multicolumn{3}{|c}{$\Gamma_{h}^{n \uparrow}(\mathrm{MeV})$} \\
\hline$\left(1 f_{5 / 2}\right)_{\mathrm{V}}$ & 2.26 & 0.05 & 0.04 \\
$\left(2 p_{1 / 2}\right)_{\mathrm{V}}$ & 0.39 & 2.65 & 0.09 \\
$\left(2 p_{3 / 2}\right)_{\mathrm{V}}$ & 0.02 & 0.20 & 0.71 \\
\hline \hline
\end{tabular}

TABLE II: Estimates for the mean values of the escape widths for the $P D R$.

\begin{tabular}{ccc}
\hline \hline $\bar{\varepsilon}_{n}-\frac{1}{2} i \bar{\Gamma}_{n}^{\uparrow}(\mathrm{MeV})$ & hole & $\bar{\Gamma}_{h}^{n \uparrow}(\mathrm{MeV})$ \\
\hline $8.6-i 1.3$ & $\left(1 f_{5 / 2}\right)_{\mathrm{v}}$ & 0.7 \\
\hline & $\left(2 p_{1 / 2}\right)_{\mathrm{v}}$ & 1.6 \\
\hline & $\left(2 p_{3 / 2}\right)_{\mathrm{v}}$ & 0.2 \\
\hline \hline
\end{tabular}

escape width is associated with the contribution of all allowed unbound particles coupled to their respective singleholes. The partial escape width for each single-hole can be approximate for $\Gamma_{h}^{n \uparrow} \simeq \sum_{p}\left|X_{p h}^{n}\right|^{2} \Gamma_{p}$, what gives a good estimate of the escape width composition for the population of several single-holes in the residual nucleus.

The single-particle energies are evaluated by solving the Schrödinger equation with Woods-Saxon potential, including the centrifugal and Coulomb (as a uniformly charged sphere) terms [3,4]. The RPA calculation is done by utilizing the Landau-Migdal residual interaction with density dependent parameters. Since the neutron and proton densities are too different in exotic nuclei, it is more appropriate to separate the nucleon density into neutron and proton parts, $\xi(r)=\frac{N}{A} \xi_{v}(r)+\frac{Z}{A} \xi_{\pi}(r)$, where each part is given by $\xi_{k}(r)=\frac{1}{1+e^{\left(r-R_{k}\right) / a_{k}}}$, with $k=\mathrm{v}(\pi)$ for neutron (proton) [2].

In this same sense, the radial single-particle orbits are represented by harmonic oscillator radial wave functions with different size parameters for neutrons and protons, $b_{k}^{2} \approx$ $\frac{4}{(3)^{\frac{4}{3}}}\left\langle r^{2}\right\rangle_{k}\left(X_{k}\right)^{-\frac{1}{3}}$, where $X_{k}=N(Z)$ for $k=v(\pi)$, and $\left\langle r^{2}\right\rangle_{k} \approx$ $\frac{3}{5} R_{k}^{2}+\frac{7}{5} \pi^{2} a_{k}^{2}[2]$. 
TABLE III: Partial escape widths for one neutron and one proton direct decay from $I S G D R$ in ${ }^{208} \mathrm{~Pb}$ nucleus. Only the main values greater than $0.1 \mathrm{MeV}$ are displayed.

\begin{tabular}{ccccccc}
\hline \hline $\bar{\varepsilon}_{n}-\frac{1}{2} i \bar{\Gamma}_{n}^{\uparrow}(\mathrm{MeV})$ & hole & $\Gamma_{h}^{\uparrow}(\mathrm{MeV})$ & hole & $\Gamma_{h}^{\uparrow}(\mathrm{MeV})$ & hole & $\Gamma_{h}^{\uparrow}(\mathrm{MeV})$ \\
\hline $24.40-i 1.35$ & $\left(2 f_{7 / 2}\right)_{v}$ & 0.58 & $\left(1 g_{7 / 2}\right)_{v}$ & 0.25 & $\left(1 h_{11 / 2}\right)_{\pi}$ & 0.19 \\
\hline$\left(1 h_{9 / 2}\right)_{v}$ & 0.47 & $\left(1 g_{9 / 2}\right)_{v}$ & 0.22 & $\left(2 d_{5 / 2}\right)_{\pi}$ & 0.13 \\
\hline$\left(2 d_{3 / 2}\right)_{v}$ & 0.19 & $\left(2 d_{5 / 2}\right)_{v}$ & 0.16 & $\left(1 g_{7 / 2}\right)_{\pi}$ & 0.23 \\
\hline \hline
\end{tabular}

\section{RESULTS AND DISCUSSIONS}

In this section, we present and discuss some results which we have obtained by using $1 p-1 h$ continuum $R P A$ approach, as described in the previous section, for isovector dipole electric transition for ${ }^{60} \mathrm{Ca}$ and isoscalar dipole transition for ${ }^{208} \mathrm{~Pb}$.

According to previous $R P A$ calculations for ${ }^{60} \mathrm{Ca}$ [5-8], our calculations [2] also predict a considerable strength in the energy region around $\sim 8.6 \mathrm{MeV}$ with a broad neutron peak. The broad width is due to the fact of the energy of the resonance to be above of the small neutron separation energy, implicating a strong coupling of the external neutrons to the continuum region. These low-lying energy states are the natural candidates for $P D R$ because they have a dominant contribution of 'neutron skin' ( $p f$ shell), in agreement with hydrodynamic interpretation. The wide peak in $\sim 8.6 \mathrm{MeV}$ is composed by the overlap of three main peaks (see TABLE I and II) exhausting about $8 \%$ of the EWSR.

In relation to the isoscalar $G D R$ in ${ }^{208} \mathrm{~Pb}$, the experimental data are not well defined and present some discrepancy.
There are some discussions in the recent literature in relation to its correct location and their widths [9-12]. Our calculations predict a considerable strength in the energy region above $20 \mathrm{MeV}$, which is composed by the presence of various narrow peaks superposing to exhaust about $82 \%$ of the EWSR between $20-30 \mathrm{MeV}$. These peaks are mainly composed by $3 \hbar \omega$ transitions involving the neutrons and protons of the externals shells. The strength of this excitation is separated in two main components, in agreement with all experimental data. The lower-energy component is due to the remaining isovector contribution. The higher-energy and broader component is identified as isoscalar GDR. The mean energy centroid and escape width of this component are $24.4 \mathrm{MeV}$ and $2.7 \mathrm{MeV}$, respectively. The partial escape widths for this mode are presented in TABLE III.

\section{ACKNOWLEDGMENTS}

This work was supported in part by Conselho Nacional de Desenvolvimento Científico e Tecnológico (CNPq), Brazil.
[1] N. Teruya, A.F.R. de Toledo Piza, and H. Dias, Nuclear Physics A 556, 157 (1993).

[2] T. N. Leite and N. Teruya, Eur. Phys. J. A 21, 369 (2004).

[3] N. Teruya, A.F.R. de Toledo Piza, and H. Dias, Phys. Rev. C 44 537 (1991).

[4] T. N. Leite, N. Teruya, and H. Dias, Int. J. Mod. Phys. E, 11, 469 (2002)

[5] F. Catara, E. G. Lanza, M. A. Nagarajan, and A. Vitturi, Nuclear Physics A 624, 449 (1997).

[6] I. Hamamoto, H. Sagawa, X. Z. Zhang, Phys. Rev. C 57 R1064 (1998).

[7] D. Vretenar, N. Paar, P. Ring, and G. A. Lalazissis, Nuclear
Physics A 692, 496 (2001).

[8] N. D. Dang, T. Suzuki, and A. Arima, Phys. Rev. C 61, 064304 (2000); N. D. Dang, V. Kim Au, T. Suzuki, and A. Arima, Phys. Rev. C 63044302 (2001).

[9] B. F. Davis et. al., Phys. Rev. Lett. 79, 609 (1997).

[10] H. L. Clark et. al. Nuclear Physics A 649 (1999) 57c-60c; H. L. Clark, Y. W. Lui , and D. H. Youngblood, Phys. Rev. C 63, 031301(R) (2001).

[11] M. Uchida et. al., Physics Letters B 557, 12 (2003).

[12] M. Hunyadi et. al., Physics Letters B 576, 253 (2003); Nucl. Phys. A 731, 49 (2004). 